\title{
De la variación morfosintáctica y otros demonios
}

\author{
La alternancia del imperfecto del subjuntivo en el español de \\ América desde una aproximación diacrónica
}

\section{Introducción}

En 1995 aparecieron dos publicaciones que han señalado las ideas fundantes de lo que se conoce como Etnopragmática en el marco de los estudios variacionistas: "Frecuencia (relativa) de uso como síntoma de estrategias etnopragmáticas" de Érica García y "Variación lingüística y Etnopragmática: dos caminos paralelos" de Angelita Martínez. Con estos textos se materializa la propuesta teórica y se inician distintas investigaciones en esta línea.

El interés por el análisis de los usos lingüísticos retoma la relación lenguacultura y, desde esta perspectiva, se incorpora a los estudios variacionistas, tal como lo propone Martínez:

La Etnopragmática intenta descubrir valores culturales a través de opciones lingüísticas que los hablantes seleccionan en diferentes contextos. Se interesa por la búsqueda de las relaciones lengua-cultura, lengua-visión del mundo a partir de dos enfoques que no se excluyen: i. cómo los factores culturales se traducen - plasman - en el uso - y sistema - del lenguaje y ii. cómo utilizamos el lenguaje para conformar contextos culturales (Martínez 1995: 427).

El análisis etnopragmático procura explicar los procesos cognitivos que subyacen a la selección que realiza el hablante, esto significa que resulta central indagar en el modo en que el desvío en la frecuencia relativa de uso de las formas revela perspectivas cognitivas (García 1995: 57). En el uso variable cobra principal interés descubrir qué contexto pragmático favorece qué forma lingüistica ya que son los contextos de aparición los que podrán explicar la presencia de las formas (García 1995: 70).

Agradecimientos: Agradezco a la Dra. Angelita Martínez la generosa lectura de este trabajo y sus atinados comentarios. Los errores son mi responsabilidad.

Adriana Speranza, Universidad Nacional de Moreno/CIC Universidad Nacional de La Plata, paglispe@gmail.com, asperanza@unm.edu.ar

¿ Open Access. (C) 2021 Adriana Speranza, published by De Gruyter. (cc) BY-NC-ND This work is licensed under the Creative Commons Attribution-NonCommercial-NoDerivatives 4.0 International License.

https://doi.org/10.1515/9783110701364-013 
Con nuestro trabajo pretendemos contribuir a esta línea de análisis aproximándonos al uso de las formas del imperfecto del subjuntivo en el español de la Argentina desde una perspectiva diacrónica. En esta ocasión, nos detendremos en algunos usos en el actual territorio argentino durante el siglo XVII. Entendemos que esta variación se relaciona con la evidencialidad como sustancia semántica que subyace a la elección del hablante. Partimos de la presunción de que el sostenimiento de este uso forma parte de las estrategias desarrolladas con el fin de expresar la evaluación del hablante acerca de la fuente de la cual obtuvo la información y acerca de la información misma, en relación con las características del fenómeno tal como se describe en la bibliografía especializada.

En este artículo presentamos, en primer lugar, el problema y los objetivos de nuestra investigación (§2). A continuación, describimos el corpus utilizado y exponemos brevemente los supuestos teóricos desde los cuales partimos (§3); luego ofrecemos una síntesis de los antecedentes sobre el tema (§4). Seguidamente nos enfocamos en el análisis de los datos (§5) y finalizamos con algunas conclusiones preliminares (§6).

\section{El problema}

Este trabajo tiene como objetivo una aproximación diacrónica al uso variable de las formas del pretérito imperfecto del modo subjuntivo, -ra y -se, en algunas variedades del español de la Argentina en documentos pertenecientes al siglo XVII. Ilustramos con el siguiente ejemplo:

1. [. . . ] porque ni avn/a ellos los rrespetan ni tienen rrespeto, ni obidiençia/a sus curacas y alcaldes, ni ellos tienen/capacidad para hazerse rrespetar y son tan grandes/borrachos como los mesmos indios, y antes/que el dicho oydor y uisitador viniera y les dejasse/en la libertad que al presente tienen, auiendo/ españoles entre ellos, estauan más domésticos/y se euitauan las borracheras e ydolatrías y otras/cerimonias que vsauan y vssan en desseruicio/de Dios Nuestro Señor, e rrespetauan a los clérigos/e frailes que los dotrinavan. . . (Argentina, año 1613, documento administrativo, CORDIAM).

Las gramáticas de uso describen la alternancia entre la forma -ra y la forma -se como distribucionalmente distinta en el español americano actual con respecto al español peninsular. Se sostiene que el español americano manifiesta una preferencia por la forma -ra, aunque la forma -se aparece en la lengua escrita (NGLE 
2010: 457). Sin embargo, algunos trabajos recientes muestran la misma tendencia en el español peninsular del siglo xx (Lara Bermejo 2019).

En lo que respecta al español americano actual, hemos realizado trabajos sobre textos literarios de autores argentinos representantes de diferentes variedades del español, correspondientes a la segunda mitad del siglo xx. El análisis inicial ha dado como resultado una distribución muy polarizada hacia la forma -ra. Hemos tomado en una primera aproximación las novelas Diario de la guerra del cerdo de Adolfo Bioy Casares (99 \% -ra y $1 \%$-se) y La traición de Rita Hayworth de Manuel Puig (97 \% -ra y 3 \% -se); en ambos casos los autores son representantes de la variedad rioplatense. A partir de estos resultados, hemos ampliado la exploración lo que nos permitió la incorporación de otros textos en los que se observa una frecuencia de aparición más alta de la forma en -se, tal como se expresa en la siguiente tabla (Speranza 2018):

Tabla 1: Distribución de las frecuencias en corpus literario de la Argentina. Segunda mitad del siglo xx (Speranza 2018).

\begin{tabular}{llrrrrrr}
\hline Región de la & Obras literarias & \multicolumn{3}{c}{ Formas en variación } & \multicolumn{2}{l}{ Totales } \\
\cline { 3 - 6 } Argentina & & \multicolumn{2}{c}{-ra } & \multicolumn{2}{c}{-se } & & \\
\hline Nordeste & Leandro Montes & 109 & $93 \%$ & 8 & $7 \%$ & 117 & $100 \%$ \\
\hline Río de la Plata & Sudeste & 241 & $79 \%$ & 66 & $21 \%$ & 307 & $100 \%$ \\
\hline Noroeste & El cantar del profeta y el bandido & 128 & $75 \%$ & 43 & $25 \%$ & 171 & $100 \%$ \\
\hline Litoral & Cicatrices & 178 & $67 \%$ & 86 & $33 \%$ & 264 & $100 \%$ \\
\hline Totales & & 656 & $76 \%$ & 203 & $24 \%$ & 859 & $100 \%$ \\
\hline
\end{tabular}

El corpus diacrónico, en cambio, muestra que la forma -se ha tenido un número mayor de ocurrencias que las halladas en los corpus del español actual. En el segmento seleccionado para este trabajo, sobre un total de aproximadamente 38000 palabras, hemos hallado 200 formas en imperfecto: 157 (77,5\%) formas en -se y 43 $(22,5 \%)$ formas en -ra. Por su parte, hemos seleccionado para el siglo XxI, a modo de muestra, un texto perteneciente al discurso jurídico: el Fallo Judicial sobre la “Tragedia de Once” publicado el 30 de marzo de 2016 por la Cámara Nacional en lo Criminal y Correccional Federal de la República Argentina. ${ }^{1}$ Del Fallo mencionado hemos cuantificado el apartado "Declaraciones indagatorias" que contiene aproximadamente 34000 palabras. Este segmento del documento presenta 98 formas del pretérito imperfecto: 90 formas en -ra (92\%) y 8 formas en -se (8\%).

1 El accidente ferroviario de la estación Once de la línea Sarmiento, comúnmente llamado Tragedia de Once, fue un siniestro ocurrido el miércoles 22 de febrero de 2012. En este accidente fallecieron 51 personas y más de 700 resultaron heridas. 
Tabla 2: Distribución de las formas en dos periodos del español americano.

\begin{tabular}{lrrrrrr}
\hline Corpus & \multicolumn{3}{c}{ Formas en variación } & \multicolumn{2}{c}{ Totales } \\
\cline { 2 - 5 } & \multicolumn{2}{c}{-ra } & \multicolumn{2}{c}{-se } & & \\
\hline Siglo $x V I I$ & 43 & $22,5 \%$ & 157 & $77,5 \%$ & 200 & $100 \%$ \\
\hline Siglo $x$ XI & 90 & $92 \%$ & 8 & $8 \%$ & 98 & $100 \%$ \\
\hline Totales & 133 & $45 \%$ & 166 & $55 \%$ & 298 & $100 \%$ \\
\hline
\end{tabular}

La comparación presentada entre dos tipos de textos homologables pretende mostrar las diferencias distribucionales entre dos estados de lengua lo que nos permitirá sustentar nuestro supuesto por el cual nos encontramos ante un cambio lingüístico en proceso. Como vemos, ambas formas se mantienen hoy en variación aunque con distribuciones diferentes de las observadas en los siglos anteriores. Los datos obtenidos sobre el siglo XVII resultan similares a los hallados en otras investigaciones correspondientes al español de la Banda Oriental para los siglos XVIII y primera mitad del XIX (Bertolotti 2000; Ramírez Luengo 2001).

Retrospectivamente, nos interesa aproximarnos al problema para entender el cambio lingüístico iniciado. Aún con las diferencias señaladas en términos sincrónicos, creemos que esta variación persiste por el aporte que el significado de cada una de las formas realiza a los mensajes que las contienen.

\subsection{Objetivos}

El objetivo general de nuestra investigación es indagar sobre el inicio del cambio a partir de las diferencias en las frecuencias halladas y cotejarlas con los corpus de las distintas zonas de América lo que podría ofrecernos indicios sobre la conformación de variedades dialectales.

Por su parte, los objetivos específicos son, en primer lugar y como hemos anticipado, aproximarnos desde una perspectiva diacrónica al uso variable del pretérito imperfecto del modo subjuntivo, -ra y -se, en la variedad del español del actual territorio de la Argentina en documentos pertenecientes al siglo XVII.

En segundo lugar, establecer la relación entre la selección de las formas y los contextos de uso. Entendemos que la posibilidad de explicación se encuentra directamente relacionada con la identificación de las motivaciones contextuales de las distribuciones halladas en el periodo seleccionado y el significado de las formas en variación, -ra y -se, significado único e invariable capaz de contribuir a la construcción de distintos mensajes, como hemos dicho. 


\section{El corpus}

Los textos sobre los que hemos trabajado integran el corpus electrónico de la Academia Mexicana de la Lengua Corpus Diacrónico y Diatópico del Español de América (CORDIAM) (www.cordiam.org). Este corpus contiene documentos recabados de archivos, de los cuales hemos seleccionado hasta el momento aquellos correspondientes a tres secciones específicas: documentos jurídicos, administrativos y documentos entre particulares (cartas y otros). Los textos administrativos contienen descripciones y lineamientos detallados de la vida cotidiana, dan cuenta de bienes materiales, de vivos y difuntos, dan testimonio de la genealogía de los individuos y de su lugar y fecha de nacimiento; mientras que los textos jurídicos corresponden a textos del ámbito legal, se acercan, en algunos aspectos, a los documentos administrativos. A diferencia de estos, sin embargo, son textos complejos y heterogéneos, ya que se componen de diferentes tipos textuales (denuncias, querellas, interrogatorios, sentencias, entre otros). Algunos de estos tipos textuales suelen ser altamente dialógicos y se aproximan, por ello, a la oralidad. En lo que respecta a los documentos particulares, hemos seleccionado una serie de cartas que, si bien son producto de la comunicación entre particulares, integran un conjunto de documentos probatorios de algún tipo de proceso. Muestran un carácter más personal sin alcanzar, en los casos analizados, las características de las cartas íntimas. Sin embargo, muestran inmediatez comunicativa porque "cartas, notas, recados, etc. son el único tipo de documento que se atreve a escribir quien no sabe 'escribir”' (CORDIAM).

\section{Variación lingüística y evidencialidad}

\subsection{Sobre la variación morfosintáctica}

El interés por los fenómenos de variación radica en la importancia que estos adquieren para el conocimiento de un determinado estado de lengua y el cambio lingüístico en progreso. Lejos de manifestar una vacilación libre o azarosa del hablante, la alternancia explicita la necesidad del usuario - en términos comunicativos - de precisar, de ajustar las "piezas” del "engranaje” lingüístico con el objetivo de crear mensajes que se acerquen a su intención. Ese esfuerzo que significa la búsqueda de un mayor rédito comunicativo se vincula con procesos cognitivos implícitos en el uso del lenguaje. Estos procesos impulsan a los hablantes a desarrollar su capacidad creativa en directa relación con las potencialidades de la propia lengua. En esta línea, partimos de la potente idea de que la sintaxis no es arbitraria, sino esencialmente motivada: “. . .las unidades lingüísticas complejas están necesariamente 
motivadas [. . .] Solo mediante estrategias que 'icónicamente' motiven las construcciones lingüísticas pueden los hablantes hacer frente, exitosamente, a la necesidad de transmitir mensajes nuevos e imprevisibles" (García 1998: 5).

Por otra parte, partimos del convencimiento de que las formas en variación poseen un significado único, invariable e impreciso que se mantiene estable en todos los contextos en los que la forma aparece. Como gran hipótesis del hablante, el significado de las formas en variación se torna la clave para entender la selección hallada. Desde esta posición, a través de dos o más formas lingüísticas en variación un evento puede representarse desde diferentes perspectivas, lo que supone "dos maneras distintas de remitir al mismo referente" (García 1985) y que dos o más términos son referencialmente equivalentes (Martínez 2000).

Desde esta perspectiva, entender la presencia de los usos alternantes requiere de una explicación. Para ello, debemos descubrir cuáles son los factores que subyacen a la selección que realiza el hablante. En este punto, tomamos distancia de la lingüística laboviana puesto que es tarea del investigador descubrir cuáles son los contextos que favorecen las formas halladas y proponer - motivar - los factores que permitirán entender la distribución de las formas a partir de la congruencia entre su aparición y los contextos en los que éstas ocurren (García 1988, García 1995; Martínez 2000, Martínez 2009). La cuantificación se torna, en este punto, un elemento central del análisis. La medición de la frecuencia relativa de uso se transforma en un "síntoma" (García 1995) que el investigador debe reconocer como el principio del proceso analítico puesto que el desvío en la frecuencia relativa de uso de las formas revela perspectivas cognitivas (García 1995, García 2009; Martínez 1995, Martínez 2000, Martínez 2010; Mauder 2001). En resumen, la variación morfosintáctica posee una motivación relacionada con la mayor compatibilidad comunicativa entre las unidades gramaticales en cuestión y el contexto léxico o sintáctico en que ocurren (García 1985: 199).

\subsection{La evidencialidad a través del análisis de la variación lingüística}

Como hemos mencionado, la variación estudiada se relaciona con la evidencialidad como sustancia semántica que subyace a la elección del hablante. Partimos de la presunción de que la alternancia de estas formas integra un conjunto de estrategias discursivas desarrolladas con el fin de expresar la evaluación del hablante acerca de la fuente de la cual obtuvo la información y acerca de la información misma.

Como hemos propuesto en trabajos anteriores (Speranza 2014), el término evidencialidad se relaciona con la forma en que distintas lenguas manifiestan 
la modalidad epistémica, enfatizando el análisis de los recursos morfológicos, léxicos o sintácticos empleados para indicar de qué manera el hablante ha tenido acceso a la información que transmite y qué evaluación hace de la misma.

En el marco de los estudios sobre el tema, Guentchèva $(1994,1996)$ propone la denominación de mediativo para la descripción de fenómenos como los citados, ya que no centra su atención en la noción de “evidencia”. Desde esta perspectiva, numerosas lenguas tipológicamente diferentes poseen formas gramaticales más o menos específicas para indicar las funciones del mediativo. A través de estos elementos, el locutor puede mostrar la distancia que toma respecto de las situaciones descritas en el evento.

En las lenguas que poseen un sistema gramatical específico de este tipo, el enunciador marca formalmente en la emisión su compromiso o distanciamiento respecto de los hechos enunciados sin por eso pronunciarse sobre su contenido referencial. De acuerdo con esta concepción, los valores fundamentales sobre los cuales se organiza el funcionamiento del mediativo son: hechos relatados, inferidos o de sorpresa. En función de las características de nuestro corpus, nos interesa concentrarnos en los dos primeros valores citados.

Los "hechos relatados" adquieren un valor particular que debe distinguirse de las formas del discurso indirecto (Guentchèva 1994: 12). El discurso indirecto constituye una enunciación citada y por lo tanto, un acto de habla que remite a una situación de enunciación que corresponde al discurso citado. El enunciado que surge del mediativo es una proposición independiente y, en aquellas lenguas que poseen codificación gramatical de esta categoría existen distinciones formales entre el mediativo y el discurso indirecto.

En lo que respecta a los "hechos inferidos", en el marco de esta propuesta teórica, se trata de una inferencia por abducción. ${ }^{2}$ La inferencia por abducción representa una reconstrucción de la situación de enunciación: el acontecimiento mediatizado es reconstruido sobre la base de las huellas observadas las cuales pertenecen a un referente distinto del referente enunciativo. El procedimiento resultante es la verbalización de un acontecimiento reconstruido y no el estado constatado (Guentchèva 1994).

Esta perspectiva resulta pertinente para abordar la alternancia propuesta ya que nos encontramos con hechos relatados y hechos inferidos. En este tipo de construcciones, el enunciador alude a información que puede haber obtenido de distintas fuentes, sin que éstas aparezcan necesariamente explicitadas, y manifiesta su evaluación sobre el contenido del enunciado.

2 Guentchèva (1994) propone la noción de inferencia abductiva siguiendo a Ch. Peirce (1965). 


\section{Antecedentes}

\subsection{Los tiempos verbales del modo subjuntivo}

Según las distintas gramáticas del español, las formas del subjuntivo son utilizadas en emisiones que exponen acciones dudosas, posibles, necesarias o deseadas (Gili Gaya 1964: 133) es decir, acciones que indican un grado menor de certidumbre puesto que su aparición se encuentra relacionada con la mayor o menor oportunidad de realización otorgada por el hablante a los acontecimientos contenidos en la emisión. La noción de certidumbre se relaciona con la expresión de las conceptualizaciones que el individuo realiza de los acontecimientos sobre los cuales posee datos suministrados por varias fuentes (Achard 2000: 163). Las formas de este modo aparecen fuertemente vinculadas a sus contextos de aparición y a la evaluación que el sujeto realiza de los acontecimientos expresados en la emisión, como hemos dicho.

Desde otra perspectiva, se sostiene para el latín - relación que nos interesa en función del significado básico que proponemos en este trabajo - que las cuatro distinciones morfológicas del modo subjuntivo indican diferencias en el tiempo en el que se desarrolló la acción y entre el tiempo y la evaluación o probabilidad otorgada por el hablante. En este punto radica la mayor complejidad del modo subjuntivo. La interacción entre estos aspectos es el factor que permite entender el cambio que puede sufrir nuestra evaluación con el paso del tiempo. El uso de las formas del subjuntivo puede indicar diferencias en el tiempo y además, diferencias en el grado de probabilidad como resultado de la interacción mencionada (Diver 2012: 185).

Según De Jonge (2004), "el subjuntivo indica que hay una alternativa relevante en el contexto, independientemente de la situación real del evento en cuestión” (2004: 207). La presencia del modo subjuntivo en la emisión, entonces, está dada no ya por la "no aserción" que habitualmente se atribuye a este modo, sino por la relevancia contextual que adquiere su utilización como "alternativa" a la ocurrencia expresada por el verbo (De Jonge 2004).

\subsubsection{El Pretérito Imperfecto}

Como hemos dicho, los tiempos del subjuntivo aparecen fuertemente vinculados a sus contextos de aparición y a la evaluación que el sujeto realiza de los acontecimientos expresados en la emisión, según las gramáticas de uso. Las formas del PI (-ra y -se) poseen correspondencia con tres tiempos del modo indicativo: pretérito perfecto simple, pretérito imperfecto y condicional simple. Comparten 
con el condicional simple del modo indicativo la propiedad de no especificar la relación temporal entre la situación designada y el momento de la enunciación por lo cual la situación puede ser anterior, simultánea o posterior al momento del habla (NGLE 2010: 458). En la utilización del PI, los límites temporales resultan poco claros. Tal como sostiene Gili Gaya (1964: 176-178), el uso del PI corresponde principalmente a la expresión del pasado y del futuro hipotético de Indicativo.

\subsubsection{El significado básico de las formas}

Dado el carácter de menor certidumbre atribuido a las acciones verbales expresadas en subjuntivo, el PI manifiesta una menor oportunidad de realización aún respecto de otros tiempos correspondientes a este modo, menor posibilidad de ocurrencia de la acción contenida en el lexema verbal, es decir, estas formas señalan una menor "posibilidad epistémica" de realización de la acción asignada por el hablante (Martínez et al. 1998). Sin embargo, como hemos anticipado, hacia el interior del PI, la forma -ra manifiesta un grado de mayor oportunidad de realización en relación con la forma -se, que indica un menor nivel de oportunidad de ocurrencia. La asignación de estos significados está dada porque las formas del PI mantienen su significado etimológico: el origen indicativo de -ra permite su incorporación en contextos menos favorables para la forma -se, propiamente subjuntiva, derivada del modo verbal latino.

\section{Los datos}

Para este trabajo, hemos consultado 15 documentos, con un total aproximado de 38000 palabras, correspondientes al siglo XVII, entre los años 1602 y 1690, pertenecientes a la región cultural de Montaña del actual territorio argentino, siguiendo la propuesta de Martínez Sarasola (1998). Esta región incluye la zona conocida tradicionalmente como Noroeste y Sierras Centrales. El corpus consultado de esta región pertenece a los actuales topónimos Jujuy, Salta, Santiago del Estero y Córdoba.

En el siglo XVI, entre las culturas originarias que habitaban la zona del Noroeste podemos citar: Omaguacas, Diaguitas, Atacameños y Comechingones. El siglo XVII se caracterizó por constituir el fin de la resistencia indígena del Noroeste. Las culturas originarias se vieron sometidas a nuevas formas de organización comunitaria, de trabajo y de mestizaje que dieron lugar a la matriz hispano-indígena cuyo desarrollo se prolongó a lo largo de los siglos posteriores. Se sumó la aparición de la etnia Colla, síntesis de diaguitas y omaguacas, y de los grupos quechua y aimara 
procedientes de Bolivia. La presencia colla se consolidó durante el siglo XIX y se constituyó en la portadora de la cultura andina (Martínez Sarasola 1998: 315-316).

Los datos anteriores ofrecen el marco general que nos permite postular el mestizaje, la convivencia de distintos grupos étnicos y lingüísticos como uno de los factores subyacentes a las diferencias distribucionales entre las variedades del español americano y el español peninsular como producto del contacto/conflicto entre lenguas y culturas. Por ello, hemos segmentado nuestro corpus en "criollos" y "no criollos" de acuerdo con el origen de los autores de los documentos a partir de los datos que en ellos aparecen. La categoría "no criollos" muestra una subdivisión entre aquellos autores identificados como españoles y aquellos casos en los que no se determina el autor. Este grupo es importante dado que aparece rotulado como "varios" sin identificación de los autores razón por la cual no podemos afirmar con certeza su origen y por este motivo, no integran nuestro análisis. La distribución observada a partir de la segmentación muestra una interesante modificación en las distribuciones a favor de la forma -ra en el corpus correspondiente a "criollos", tal como se observa en la tabla que sigue:

Tabla 3: Distribución de las formas según origen de los autores siglo XVII.

\begin{tabular}{lrlrrrrr}
\hline \multirow{2}{*}{ Corpus } & \multicolumn{4}{c}{ Formas en variación } & \multirow{2}{*}{ Totales } \\
\cline { 2 - 6 } & & \multicolumn{2}{l}{-ra } & \multicolumn{2}{c}{-se } & & \\
\hline Criollos & 29 & $41 \%$ & 41 & $59 \%$ & 70 & $100 \%$ \\
\hline \multirow{2}{*}{ No criollos } & Españoles & 5 & $23 \%$ & 17 & $77 \%$ & 22 & $100 \%$ \\
\cline { 2 - 7 } & Varios & 9 & $8 \%$ & 99 & $92 \%$ & 108 & $100 \%$ \\
\hline Totales & 43 & $22.5 \%$ & 157 & $77.5 \%$ & 200 & $100 \%$ \\
\hline
\end{tabular}

Distintos autores que han descrito de manera exhaustiva el tema (Cano Aguilar 2014; Lapesa 1981; Lara Bermejo 2019; Veiga 2006, entre otros) consideran el siglo XVII como el momento de consolidación de la forma -ra como propia del subjuntivo:

Como es sabido, en los siglos XVI y XVII se consuman dos de los cambios más trascendentes para la historia del subjuntivo español, que llevan a una notable simplificación del sistema heredado de la lengua medieval: por un lado, el desuso, pero no desaparición completa, de la forma en -re en prácticamente todos sus contextos [. . . ] por otro, el cambio de valores de -ra, que pierde progresivamente su marca de "irreal”, y su referencia al pasado, para igualarse en casi todos los usos con -se, generando así una situación curiosa en los sistemas verbales: la coexistencia de dos formas con prácticamente los mismos valores, sin que una de ellas sea sacrificada de forma inmediata (Cano Aguilar 2014: 3940). 
En esa trayectoria de expansión, distintos trabajos muestran que las estructuras condicionales han sido los contextos privilegiados para -ra (Cano 2014; Veiga 2006). Dada esta afirmación, hemos medido en nuestro corpus, tanto para criollos como para españoles, la distribución de las formas en relación con el tipo de estructura, tal como aparece en la siguiente tabla:

Tabla 4: Distribución de las formas según el tipo de estructura sintáctica y corpus, siglo XVII.

\begin{tabular}{|c|c|c|c|c|c|c|c|c|}
\hline \multirow[t]{3}{*}{ Estructuras } & \multicolumn{4}{|c|}{ Corpus criollos } & \multicolumn{4}{|c|}{ Corpus españoles } \\
\hline & \multicolumn{4}{|c|}{ Formas en variación } & \multicolumn{4}{|c|}{ Formas en variación } \\
\hline & \multicolumn{2}{|c|}{-ra } & \multicolumn{2}{|c|}{-se } & \multicolumn{2}{|r|}{-ra } & \multicolumn{2}{|r|}{-se } \\
\hline Condicionales & 12 & $63 \%$ & 7 & $37 \%$ & 2 & $29 \%$ & 5 & $71 \%$ \\
\hline Sustantivas & 4 & $15 \%$ & 22 & $85 \%$ & 2 & $40 \%$ & 3 & $60 \%$ \\
\hline Comparativas & 3 & $100 \%$ & 0 & $0 \%$ & 0 & $0 \%$ & 0 & $0 \%$ \\
\hline Concesivas & 7 & $100 \%$ & 0 & $0 \%$ & 0 & $0 \%$ & 0 & $0 \%$ \\
\hline Finales & 3 & $33 \%$ & 6 & $67 \%$ & 0 & $0 \%$ & 4 & $100 \%$ \\
\hline Temporales & 0 & $0 \%$ & 1 & $100 \%$ & 0 & $0 \%$ & 2 & $100 \%$ \\
\hline Relativas & 0 & $0 \%$ & 5 & $100 \%$ & 1 & $25 \%$ & 3 & $75 \%$ \\
\hline Totales & 29 & $41 \%$ & 41 & $59 \%$ & 5 & $23 \%$ & 17 & $77 \%$ \\
\hline
\end{tabular}

Como vemos, la distribución de las formas muestra una tendencia a favor de la descripción citada, particularmente en el corpus de "criollos”. Si bien esa tendencia confirma los datos hallados en las investigaciones diacrónicas, el número de apariciones de -ra en emisiones condicionales resulta insuficiente para explicar la aparición del resto de las formas.

A partir de una observación más detallada de los materiales con los que trabajamos, intentaremos aproximarnos a una posible explicación sobre las frecuencias observadas en el corpus correspondiente a "criollos". Como hemos planteado, nuestro interés consiste en acercarnos a una explicación acerca del cambio lingüístico iniciado y de las motivaciones que subyacen al uso alternante de -ra vs. -se, en el corpus seleccionado. En esta ocasión, proponemos los siguientes parámetros:

(i) El tipo textual

(ii) La expresión del punto de vista

A continuación, exploraremos en qué medida estos factores influyen en la selección de las formas y nos permiten comprender las distribuciones halladas. 


\subsection{El tipo textual}

Si ponemos la lupa en las características de los documentos, encontraremos allí un indicio que podría ayudarnos a pensar en las razones que dan lugar al aumento de la forma -ra. De acuerdo con la descripción realizada sobre los tipos textuales, los documentos administrativos son de carácter burocrático por lo que se muestran más pautados, más normatizados, menos permeables a los cambios. Veamos un ejemplo:

2a) [... .] después de la Muerte del dicho Marido el que muriese Yo sin tener ocasion en este paraje tan despoblado de hacer otro nuevo testamento en este casso dejaua por herederos a los Padres de la Compania de Jesus de la provincia del tucuman para que fundasen un Colegio en la Villa de Tarija para la redución a Nuestra Santa fee de los Yndios chiriguanas y otras naciones Y en este dicho casso dejaua por uno de mis Albacea al Rector que al presente era o adelante fuese de la Ciudad de Salta Y por que despues de todo esto solicitaron dichos Padres que Yo y el dicho Mi Marido procurasemos en vida hacer dicha fundación (Argentina, año 1690, administrativo, CORDIAM).

Por su parte, los documentos jurídicos, aún con la diversidad textual que manifiestan, se presentan más dialógicos, más abiertos a la incorporación de formas menos esperadas, al igual que los documentos particulares - cartas y otros -, por lo tanto más cercanos a la oralidad. Ilustramos con los siguientes ejemplos:

2b) [. . . me remuerde grauemente la conçiençia de no hauer puesto otros medios mas eficaçes, aunque fuera con costa de desamparar estas ouejas ad [. . . ] y ir a la presencia de Vuestra real Persona a boluer por ellas aunque en la nauegacion pusiera a rriesgo mi vida. Hame estoruado ultimamente este medio las ultimas y christianissimas cedulas de Vuestra Magestad en que tan apretadamente manda se quite este diabolico seruicio y el orden que Vuestra Magestad se ha seruido en usar mandando al Licenciado Maldonado. .. (Argentina, año 1609, jurídico, CORDIAM).

2c) [. . . q que Oliba y otros mercaderes de ai me lo pidieron -pero mexor le sera a Vm enbiallo al Puerto -que ay mucha demanda del, que Rossillo me pago a sus reales sien libras que llevo al Puerto el otro dia, a Juanilla la india que esta en mi casa le pida Vm la plata que ubiere hecho ay del pan y otras cossas, que seran algunos quarenta u sincuenta pesos, y perdone Vm que quisiera acudir diferentemente a quien tan bien lo merese que si tubiera bueyes, pudiera aver ynbiado sien hanegas de harina y otras tantas de maiz. . . (Argentina, año s/d, cartas, CORDIAM). 
Creemos que los contextos como (2a), por su carácter menos personal y más estandarizado, favorecen la selección de -se. Estos contextos ofrecen mayor resistencia a la introducción de cualquier innovación, como hemos mencionado. Por lo tanto, la selección de esta forma, a partir de su significado básico, resulta la más propicia. Por su parte, los contextos como (2b) y (2c), por su carácter más personal, menos normatizado, más cercanos a la oralidad como hemos dicho, resultan más apropiados para el cambio, representado en este caso por la expansión de la forma -ra. La cuantificación, arroja los siguientes resultados:

Tabla 5: Distribución de las formas según el tipo textual. Corpus criollos, siglo xVII.

\begin{tabular}{lrrrrrr}
\hline & \multicolumn{2}{c}{-ra } & \multicolumn{2}{l}{-se } & \multicolumn{2}{c}{ Totales } \\
\hline Docs. particulares y jurídicos & 25 & $62,5 \%$ & 15 & $37,5 \%$ & 40 & $100 \%$ \\
\hline Docs. Administrativos & 4 & $13 \%$ & 26 & $87 \%$ & 30 & $100 \%$ \\
\hline Totales & 29 & $41 \%$ & 41 & $59 \%$ & 70 & $100 \%$ \\
\hline
\end{tabular}

o.r.: $10.83 \chi^{2}=17.08 p<0.001$

Los resultados de la cuantificación permiten verificar la asociación entre el parámetro propuesto y el significado de las formas en variación, -ra y -se. La distribución observada nos ofrece una perspectiva acerca de los posibles intersticios por los que se ha ido incorporando la forma menos esperada.

Una vez más, volvemos nuestra mirada hacia las estructuras que acogen las formas en variación. A partir de la caracterización realizada más arriba, intentaremos probar si el tipo textual favorece la expansión de la forma -ra en las estructuras condicionales. Los datos son los siguientes:

Tabla 6: Distribución de las estructuras condicionales según el tipo textual. Corpus criollos, siglo XVII.

\begin{tabular}{lrrrrrr}
\hline & \multicolumn{2}{c}{-ra } & \multicolumn{2}{c}{-se } & \multicolumn{2}{c}{ Totales } \\
\hline Docs. particulares y jurídicos & 12 & $86 \%$ & 2 & $14 \%$ & 14 & $100 \%$ \\
\hline Docs. Administrativos & 0 & $0 \%$ & 5 & $100 \%$ & 5 & $100 \%$ \\
\hline Totales & 12 & $63 \%$ & 7 & $37 \%$ & 19 & $100 \%$ \\
\hline
\end{tabular}

Verificamos cuantitativamente que la variación solo se observa en los documentos jurídicos y particulares que, como hemos sostenido, se muestran más flexibles, más dialógicos y, por lo mismo, candidatos óptimos para la expansión de la forma menos esperada. El significado indicativo pervive; ese significado permite filtrar la evaluación del enunciador, aún en estos contextos, como estra- 
tegia pragmática y argumentativa, en el mismo sentido que propone Cano Aguilar (2014) para otras condicionales.

\subsection{La expresión del punto de vista}

En la misma línea de análisis, si atendemos al contenido y características de las emisiones, la frecuencia de la forma -ra aumenta en relación con los textos que favorecen la presencia de la voz del locutor a través de declaraciones, denuncias, pedidos, entre otros.

A partir del análisis de los contextos, retomamos la noción de "punto de vista” (en adelante PdV) desarrollada en trabajos anteriores (Speranza 2014) para aproximarnos a una explicación sobre los datos. Proponemos la noción de PdV para referirnos a la perspectiva de los participantes introducidos en el discurso. Desde nuestra propuesta, los "sujetos discursivos" constituyen la fuente a la cual el enunciador otorga un nivel de "confiabilidad" por lo que a los hechos reproducidos les atribuye, a su vez, un determinado grado de factualidad y certidumbre, en relación con la responsabilidad que la fuente es susceptible de asumir desde los puntos de vista expresados en la emisión (Speranza 2014:108).

El PdV es, entonces, la representación de la perspectiva de los "sujetos discursivos”, a quienes el locutor les atribuye una intervención determinada en función de la evaluación que realiza de los hechos descritos en los eventos presentados. En los documentos analizados, los participantes del discurso poseen diferencias en función del lugar que ocupan en la escala social. Así, encontramos participantes con mayor jerarquía a quienes el locutor se dirige para solicitar su favor, informar, para sugerir, para denunciar. Estos sujetos pueden estar directamente involucrados en el desarrollo de los eventos, son responsables de la toma de decisiones, resuelven conflictos, entre otras cosas. Por otro lado, encontramos participantes con menor jerarquía en la pirámide social que por lo mismo poseen una posición menos destacada en lo que se refiere a la toma de decisiones, resolución de conflictos, etc.

En este caso, los enunciados en los que aparece de manera explícita el PdV del locutor que, en la mayor parte de los casos, ocupa un lugar menos destacado en la escala social, resultan los candidatos más propicios para la selección de la forma -ra, puesto que, desde nuestra postulación, el locutor les asignará mayor oportunidad de realización como expresión de garantía, como fuente de la información que transmite. Veamos el siguiente ejemplo:

3a) Y en quanto a la pregunta que se me/hace en qué estoy ocupada, digo que la que yo tengo es de estar/siempre en mi casa pronta y aparejada para acudir 
con el socorro/que se me pide y manda dar por los gouernadores que an sido y son/desta tierra en las cosas tocantes al rreal seruicio, donde siempre/e acudido con armas y cauallos y matalotaje que e dado al/soldado que se me echa por suerte para que le avíe como es público y/notorio, sin ser rreseruada como lo pudiera ser por ser muger/biuda, pobre y sola, y gozar de menos feudo <inter: y rrentas>, corta y muy estrecha/que todos los vezinos desta ciudad donde soy vezina, suçesora en la/segunda vida de Pedro Ximénez, mi marido, que Dios aya, ... (Argentina, año 1608, jurídico, CORDIAM).

Este ejemplo presenta un caso en el que -ra podría alternar con la forma condicional. Si bien se consideran contextos en los que la variación, en principio, no se manifiesta - cuestión que vamos a considerar así puesto que en nuestro corpus actual no disponemos de la forma -se para establecer el contraste -, entendemos que esta selección se inscribe en lo que Veiga denomina "empleos de cortesía en el caso de los verbos modales poder, deber y querer" (2006: 179). En este caso, entre las opciones del hablante y en el marco de las relaciones descritas, la selección de -ra resulta una opción intermedia entre la mayor oportunidad de realización, que podría expresarse a través del condicional, cuestión que nos abordaremos puesto que excede los objetivos de este trabajo, y la menor oportunidad expresada por la forma -se. ${ }^{3}$ Podríamos postular que la selección de esta forma a comienzos del siglo XVII marca el camino de la distribución actual hallada (Speranza 2018).

Por otra parte, los contextos en los que el locutor se dirige a personajes con poder y jerarquía, o aquellos contextos en los que describe acciones realizadas por ellos o solicitadas por la autoridad que representan, o a la que se ven subordinados, o contextos en los cuales hace recomendaciones a las autoridades resultan eventos en los que el locutor posee menos control, menor certeza ya que la efectiva realización de las acciones o su descenlace está en manos de quien ejerce la autoridad, por lo que no puede funcionar como garante y por lo mismo, se presenta más cauteloso, menos acertivo, más respetuoso. Por ello, asignará un grado menor de oportunidad de realización del evento a través de la selección de la forma -se, como aparece en el ejemplo que sigue:

3b) Facilitaria tambien la conuersion de los Infieles, que Vuestra Majestad se siruiese de mandar enuiar sobre carta de la Cedula en que se ordena, que los indios reçien conuertidos no tengan seruiçio alguno, ni paguen tassa por

3 Los análisis realizados sobre el español de la Argentina del siglo xx, particularmente en el Río de la Plata, muestran la expansión del condicional sobre el imperfecto más allá de la estigmatización manifiesta sobre esta selección (Lavandera 1984). 
diez años los quales son menester para domesticarlos, y enseñalles la justicia y doctrina christiana; y assimismo couiene prohiuir con gravissimas penas las Malocas y entradas, que no son otra cosa mas, que una monteria, y caza de indios, que luego hazen esclauos, y como tales los venden. . . (Argentina, año 1609, administrativos, CORDIAM).

Una aproximación cuantitativa nos permite corroborar nuestra postulación:

Tabla 7: Frecuencia de uso según el PdV presentado.

Corpus criollos, siglo XVII.

\begin{tabular}{lcccccc}
\hline & \multicolumn{2}{c}{-ra } & \multicolumn{2}{c}{-se } & \multicolumn{2}{c}{ Totales } \\
\hline+ PdV locutor & 19 & $73 \%$ & 7 & $27 \%$ & 26 & $100 \%$ \\
\hline - PdV locutor & 10 & $23 \%$ & 34 & $77 \%$ & 44 & $100 \%$ \\
\hline Totales & 29 & $41 \%$ & 41 & $59 \%$ & 70 & $100 \%$ \\
\hline
\end{tabular}

o.r.: $9.22 \chi^{2}=17.05 p<0.001$

La distribución observada integra una estrategia de refuerzo, a través de la selección de la forma -ra, en aquellos segmentos que permiten el despliegue de la perspectiva del locutor, su evaluación de los hechos relatados, su posición frente al evento y la construcción de su PdV como estrategia argumentativa. Por su parte, la selección de la forma -se resulta una estrategia de mitigación, contraparte de la anterior, al dirigirse a una autoridad de la cual depende y ante la cual se presenta en relación de dependencia y subordinación, todo ello sustentado por el significado de las formas.

Más allá de los resultados que, en principio, favorecen nuestra hipótesis, observamos un grupo de enunciados que aparecen como aparentes "contraejemplos”. Veamos:

Tabla 8: Aparentes contraejemplos. Corpus criollos, siglo XVII.

\begin{tabular}{lcccccc}
\hline & \multicolumn{2}{c}{-ra } & \multicolumn{2}{c}{-se } & \multicolumn{2}{c}{ Totales } \\
\hline+ PdV locutor & 19 & $73 \%$ & 7 & $27 \%$ & 26 & $100 \%$ \\
\hline- PdV locutor & 10 & $23 \%$ & 34 & $77 \%$ & 44 & $100 \%$ \\
\hline Totales & 29 & $41 \%$ & 41 & $59 \%$ & 70 & $100 \%$ \\
\hline
\end{tabular}

o.r.: $9.22 \chi^{2}=17.05 p<0.001$ 
En 7 casos aparece la forma -se en contextos en los que se presenta el PdV del locutor y en 10 casos aparece la forma -ra en contextos en los que se presenta un PdV ajeno al locutor.

En lo que respecta a las formas en -se,corresponden a 3 documentos, 2 de ellos fechados en 1608 y el tercero en 1690. En los 3 casos correspondientes a los documentos de 1608, el locutor describe acciones a partir de órdenes recibidas de sus superiores; en ellas el locutor recibe la fuerza del evento por lo que la perspectiva presentada es ajena, tal como se observa en el siguiente ejemplo:

4a) /el gouernador don Pedro de Mercado Peñalosa, que entonçes $\{\mathrm{f} .1 \mathrm{v}\}$ tenía a su cargo el gouierno d[e] ellas, /me mandó que fuese al valle de Calchaquí con la/demás gente que él lleuaua al castigo y paçificaçión/de los yndios çircunvezinos de aquel valle que estauan/alçados y alborotados por auer muerto a vn frayle francisco/y a ciertos españoles, y que convenía que yo fuese con [... . (Argentina, año 1608, cartas, CORDIAM).

Los 4 casos restantes corresponden a un documento administrativo del año 1690 en el cual se manifiesta la voluntad de una mujer por derogar y revocar ciertas cláusulas de su testamento. Este caso acompaña los datos presentados más arriba en la tabla 5 ya que es un documento administrativo, a la vez que presenta la voz de una mujer con una posición destacada en la escala social, que toma la decisión de cambiar la voluntad original de su testamento. En todos los casos, la expresión de esa voluntad se ve acompañada por el consentimiento de su marido que es, además, su heredero, tal como se verifica en el siguiente ejemplo:

4b) [...] Y porque fue condición deella que Yo la huuiese de aprouar y ratificar y pareciendome que aunque no en lo referido en otrascircunstancias me pejudicaua dicha donacion dispuse que se chancelase como se hizo Y el dicho mi Marido y Yo hicimos otra de nueuo que passo y otorgamos ante el dicho Theniente y Justicia Mayor de este dicho partido como también dicha reuocación con consentimiento y aprobación del Padre. . . (Argentina, año 1690, administrativos, CORDIAM).

Por su parte, la presencia de la forma -ra en contextos menos esperados corresponde a un documento fechado en 1613. Se trata del testimonio de un clérigo, el padre Gerónimo de Godoy contra el alguacil Rodrigo de Soria; la declaración está dirigida al Rey. El documento es una denuncia por la que se acusa a Soria de corrupción, excesos de autoridad, robo, violencia, etc. Veamos el siguiente fragmento: 
4c) ... hombre dañino perjudiçial en esta Republica Siendo contrario d[e] ella desfauoreçiendola con juezes vibiendo Con escandalo de su persona y lengua en las Cossas que haze por quanto no ay persona que este bien con el y si no fuera por el miedo que tienen de que les a de perseguir con la bara que trae de Alguaçil mayor todo el pueblo hombres y mugeres entiende y tiene por çierto este testigo juraran esto que tiene dicho y otras Cossas mas de que se a acortado dezirlas por ser Saçerdote. . . . . . ] sabe este testigo que a un feligres suyo llamado felis mulato del Seruiçio de $\{f .4\}$ Geronimo Diaz Maestro le quito quinze anegas de mayz Y muchas aues el dicho alguaçil mayor deziendo que el padre del dicho felis que auia poco que auia falleçido auia dejado orden para que se lo dixeran de missas y que el susso dicho lo tomaua a su Cargo para pagarlo al dicho felis y este testigo sabe que no se lo a pagado a mas tiempo de dos años y el dicho mulato se le a quexado a este testigo. . . (Argentina, año 1613, jurídicos, CORDIAM).

Todo el testimonio es una larga denuncia contra una autoridad ante otra autoridad mayor. En esa escala de poder, el denunciante - integrante también de esa escala jerárquica - fundamenta sus declaraciones en la evidencia obtenida a través de su experiencia, lo que lo transforma en una fuente con información de primera mano, y en la evidencia obtenida de segunda mano por medio de las declaraciones de quienes padecen la violencia y el engaño de la autoridad: “. . . este testigo como cura y beneficiado del partido de las estancias en su districto ha visto por vista de ojos y otras de oydas y quejas de sus feligreses. ..” Además, a lo largo de su extenso testimonio se encarga de destacar el conocimiento que posee de los hechos: "sabe este testigo. .." con estas precisiones pretende mostrar la validez de su discurso; discurso que se ve reforzado a través de la selección de la forma -ra con la cual otorga mayor grado de factualidad y certidumbre a las acciones descritas.

\section{Conclusiones}

En el presente trabajo hemos pretendido acercarnos a un caso de variación lingüística que integra los usos estandarizados del español actual. Hemos intentado retrotraernos al siglo XVII para comenzar a entender las diferencias distribucionales y acercarnos a las motivaciones que podrían explicar el cambio en proceso. Nos mueve el interés por encontrar la clave que inició la expansión de la forma -ra en el español de la Argentina, en el caso que nos ocupa. 
Los factores que nos permiten explicar la variación se relacionan, como hemos propuesto, con el grado de oportunidad de realización que el enunciador le otorga al contenido referencial de la emisión. Esta asignación aparece representada por las características de los textos en los que las formas aparecen y la manifestación del PdV a través de la explicitación de la evaluación, de la “doxa”, expresada en el discurso.

Desde nuestra propuesta, dentro de la menor certidumbre expresada por la morfología del subjuntivo, entre las formas del PI existe una sutil diferencia, aunque muy relevante, en cuanto a la idiosincrasia humana. La expansión y pervivencia de la forma -ra se explica por el significado etimológico de la forma. En efecto, su origen indicativo derivado del latín se ha mantenido y ha admitido su ingreso, tímido al inicio pero sostenido, en contextos menos esperados. El aporte de ese significado originario ha contribuido a la convivencia de ambas formas $\mathrm{y}$ su explotación creativa por parte de los usuarios.

La aparición de la forma -ra en contextos menos esperados integra un proceso similar al abordado en investigaciones anteriores (Speranza 2014: 185) por el cual el presente del subjuntivo toma contextos del PI. En este caso, pretendemos mostrar cómo dentro del PI, -ra avanza cuantitativamente sobre la presencia de -se y marca una tendencia que acompaña la sustitución del subjuntivo por el indicativo; tendencia que torna relevante la comparación presentada más arriba por la cual las diferencias distribucionales dan cuenta del cambio iniciado.

Por otra parte, creemos que esta alternancia, lejos de manifestar "dos formas distintas de decir lo mismo" (Labov 1983: 241), muestra el aporte que el significado básico de las formas realiza al mensaje. Entendemos que esta es la razón central por la cual la variación se ha mantenido en el tiempo incluso con las modificaciones en la distribución señaladas.

Los datos obtenidos nos han permitido verificar la vinculación entre los usos variables y los significados básicos postulados para las formas del PI. Creemos que estos significados son congruentes con el análisis realizado y nos permiten vincular los usos hallados a la evidencialidad como forma de expresión de los modos de apropiación del conocimiento y el grado de validez otorgado por el sujeto a la información que transmite el enunciado. Partimos de la presunción de que el sostenimiento de este uso forma parte de un conjunto de estrategias discursivas desarrolladas para este fin. El enunciador da cuenta de la información que transmite y de su conocimiento, de la forma en la cual la ha adquirido y cómo la evalúa, lo que significa presentarla a un potencial interlocutor como +/- factual, +/- posible, en este caso. Creemos que estos usos constituyen un reaprovechamiento de las formas del PI, de la misma manera que hemos observado 
en investigaciones anteriores sobre estas y otras formas que integran el sistema verbal del español (Speranza 2011, 2014, 2018).

\section{Corpus bibliográfico}

CORDIAM = Academia Mexicana de la lengua. Corpus Diacrónico y Diatópico del Español de América. URL: www.cordiam.org.

Fallo Judicial sobre la "Tragedia de Once" publicado el 30 de marzo de 2016 por la Cámara Nacional en lo Criminal y Correccional Federal de la República Argentina. URL: www.cij. gov.ar.

\section{Referencias bibliográficas}

Achard, Michel. 2000. Selección de modo en construcciones oracionales de complemento. Revista española de lingüística aplicada (número monográfico 1). 153-173.

Bertolotti, Virginia. 2000. El imperfecto del subjuntivo: aspectos diacrónicos y sincrónicos. Ponencias de profesores uruguayos presentadas en los congresos de la UBA y de la ALFAL, 11-17. Montevideo: Publicación de la Sociedad de Profesores de Español del Uruguay.

Cano Aguilar, Rafael. 2014. Oraciones condicionales. En Concepción Company (ed.), Sintaxis histórica de la lengua española, 3909-4091. México: FCE/UNAM.

Diver, William. 2012. The subjunctive without syntax. En Alan Huffman y Joseph Davis (eds.), Language: Communication and Human Behavior. The Linguistic Essays of William Diver, 183-193. Leiden y Boston: Brill.

García, Érica. 1985. Shifting variation. Lengua 67. 189-224.

García, Érica. 1988. Lingüística Cartesiana o el Método del Discurso. Lenguaje en Contexto 1. 5-36.

García, Érica. 1995. Frecuencia (relativa) de uso como síntoma de estrategias etnopragmáticas. En Klaus Zimmermann (ed.), Lenguas en contacto en Hispanoamérica, 51-72. Madrid y Fráncfort: Iberoamericana/Vervuert.

García, Érica. 1998. Qué cuenta, y cómo contar en lingüística. En Christian de Paepe y Nicole Delbecque (eds.), Estudios en honor del profesor José de Kock, 217-223. Lovaina: Leuven University Press.

García, Érica. 2009. The Motivated Syntax of Arbitrary Signs. Amsterdam y Filadelfia: John Benjamins.

Gili Gaya, Samuel. 1964. Curso superior de sintaxis española. 9ํeición. Barcelona : Vox. Guentchèva, Zlatka. 1994. Manifestations de la catégorie du médiatif dans les temps du français. Langue Française 102. 8-23.

Guentchèva, Zlatka. 1996. Introduction. En Guentchéva Zlatka (ed), L'Énonciation médiatisée, 11-18. París y Lovaina: Éditions Peeters.

Jonge, Bob de. 2004. The relevance of relevance in linguistic analysis. Spanish subjunctive mood. En Ellen Contini-Morava, Robert S. Kirsner y Betsy Rodríguez Bachiller (eds.), 
Cognitive and communicative approaches to linguistic analysis. Vol. 51, 206-218.

Filadelfia: John Benjamins.

Labov, William. 1983. Modelos sociolingüísticos. Madrid: Cátedra.

Lapesa, Rafael. 1981. Historia de la lengua española. Madrid: Gredos.

Lara Bermejo, Víctor. 2019. El pretérito imperfecto de subjuntivo en la Península Ibérica del siglo xx. Verba 46. 313-338.

Lavandera, Beatriz R. 1984. Variación y significado. Buenos Aires: Hachette.

Martínez, Angelita. 1995. Variación lingüística y Etnopragmática: dos caminos paralelos.

En Actas de las Segundas Jornadas de Lingüística Aborigen, 427-437. Buenos Aires: Instituto de Lingüística/Universidad de Buenos Aires.

Martínez, Angelita. 2000. Lenguaje y cultura. Estrategias etnopragmáticas en el uso de los pronombres clíticos lo, la y le en la Argentina en zonas de contacto con lenguas aborígenes. Leiden: Tesis de doctorado de la Universidad de Leiden.

Martínez, Angelita. 2009. Metodología de la investigación lingüística: el enfoque etnopragmático. En Elvira Narvaja de Arnoux (ed.), Escritura y producción de conocimiento en las carreras de posgrado, 259-286. Buenos Aires: Santiago Arcos.

Martínez, Angelita. 2010. Lenguas y variedades en contacto. Problemas teóricos y metodológicos. Revista Internacional de Lingüística Iberoamericana 15. 9-31.

Martínez, Angelita, Beatriz Gualdieri y Liliana Oberti. 1998. Alternancia y frecuencia de uso en las condicionales contrafactuales de pasado: una interpretación cualitativa. En Actas del IX Congreso Internacional de la Asociación Lingüística y Filológica de América Latina (ALFAL), 97-106. Campinas: Universidad Estatal de Campinas/Instituto de Estudios del Lenguaje.

Martínez Sarasola, Carlos. 1998. Nuestros paisanos los indios. Vida, historia y destino de las comunidades indígenas en la Argentina. Buenos Aires: Emecé.

Mauder, Elisabeth. 2001. Variación lingüística y etnopragmática. Factores socio-culturales en la variación ser y estar. Etnopragmática. Signo \& Seña 11. 223-241.

Ramírez Luengo, José Luis. 2001. Alternancia de las formas -ra/-se en el español uruguayo del siglo xıx. Estudios filológicos (Valdivia, Universidad Austral de Chile) 36. 173-186.

Real Academia Española y Asociación de Academias de la Lengua. 2010. Nueva gramática de la lengua española. Madrid: Espasa Calpe.

Speranza, Adriana. 2011. Evidencialidad en español. Su análisis en variedades del español en contacto con las lenguas quechua y guaraní en el Gran Buenos Aires y la Ciudad de Buenos Aires. Buenos Aires: Tesis doctoral de la Universidad de Buenos Aires.

Speranza, Adriana. 2014. La evidencialidad en el español americano. La expresión lingüística de la perspectiva del hablante. Madrid y Fráncfort: Iberoamericana/Vervuert.

Speranza, Adriana. 2018. Sobre tendencias gramaticales y distribuciones observadas. La alternancia del imperfecto del subjuntivo como estrategia evidencial en el español de la Argentina. Informe Posdoctoral correspondiente al Programa de Posdoctorado en Ciencias Humanas, Facultad de Filosofía y Letras, Universidad de Buenos Aires. Inédito.

Veiga, Alexandre. 2006. Las formas verbales subjuntivas. Su reorganización modo-temporal. En Concepción Company (ed.), Sintaxis histórica de la lengua española, 95-240. México: FCE/UNAM. 
